\title{
Erratum: Optical coupling of two distant InAs/GaAs quantum dots by a photonic-crystal microcavity [Phys. Rev. B 81, 193301 (2010)]
}

\author{
E. Gallardo, L. J. Martínez, A. K. Nowak, D. Sarkar, H. P. van der Meulen, J. M. Calleja,* C. Tejedor, I. Prieto, \\ D. Granados, A. G. Taboada, J. M. García, and P. A. Postigo \\ (Received 20 February 2012; published 30 March 2012)
}

DOI: 10.1103/PhysRevB.85.129903

PACS number(s): 78.67.Hc, 42.50.Ex, 78.67.De, 99.10.Cd

The coupling values $g_{1,2}$ extracted from the data in Fig. 2 using Eq. (1) are not $30 \mu \mathrm{eV}$, as stated, but $75 \mu \mathrm{eV}$. The error comes from a mistake in the fitting procedure. As a consequence, the interdot effective coupling is $J \cong 11 \mu \mathrm{eV}$, instead of $2 \mu \mathrm{eV}$. None of the conclusions is affected by this change.

*jose.calleja@uam.es 